\title{
Evolución del consumo de antibióticos y de la susceptibilidad antimicrobiana en el Centro Hospitalario Pereira Rossell en la era de Staphylococcus aureus resistente a meticilina
}

\author{
Héctor Telechea O., Noelia Speranza M., Liriana Lucas P., Adriana Santurio G., \\ Gustavo Giachetto L., Gabriela Algorta R., Luciana Nanni R. y M. Catalina Pírez G.
}

\section{Antibiotic consumption and antimicrobial susceptibility evolution in the Centro Hospitalario Pereira Rossell in methicillin resistant Staphylococcus aureus era}

Introduction: In the past few years, an increase in methicillin resistant-not multiresistant Staphylococcus aureus was observed in Uruguay among children with community acquired infections. Recommendations for empiric antibiotic treatment required adjustments and new national guidelines were recommended in July 2004. Adherence to these guidelines was indirectly performed by monitoring antibiotic consumption and antimicrobial susceptibility patterns in Uruguay. Objective: To describe and compare antibiotic consumption and antimicrobial susceptibility of Staphylococcus aureus in a Pediatric Hospital of the Centro Hospitalario Pereira Rossell (PHCHPR) between 2001 and 2006. Methods: Antibiotic consumption in hospitalized children was calculated using the Defined Daily Dose per 100 bed-days (DDD/100). Reference values were obtained from the World Health Organization Collaborating Center for Drug Statistics Methodology of. Consumption. Data were obtained using the WinPharma programme of the Pharmacy Department of CHPR. The fraction of annual occupancy of hospital beds was obtained from the Statistic Division of CHPR. Antibiotic consumption was evaluated netween 2001 and 2006 and expressed as DDD/100 and percent change. Antimicrobial susceptibility was evaluated using CHPR's Microbiology Laboratory data during the same time period. Results: After 2003 a significant increase in consumption of clindamycin, ceftriaxone, trimethoprim-sulphamethoxazole, cefuroxime, vancomycin and gentamycin was observed, except for cephradine. Consumption of clindamycin, ceftriaxone and trimethoprim-sulphamethoxazole showed the highest increase $(6.15 \% ; 1.44 \%$ and $1.17 \%$ respectively). Detection of Staphylococcus aureus increased significantly mostly from skin and soft tissue infections. Oxacillin susceptibility of S aureus strains obtained from different sites had a significant and persistent decrease after 2003 (from $81 \%$ during year 2001 to $40 \%$ in year 2006 $(\mathrm{p}<0.05)$. Susceptibility to others antibiotics did not decrease. Between 2004 and 2006 the "D effect" decreased from $28 \%$ to $21 \%$. Antimicrobial susceptibility patterns did not differ by site of infection. Conclusions: Methicillin resistant-not multiresistant Staphylococcus aureus has established itself as a regular community pathogen in Uruguayan children. Changes in antimicrobial consumption patterns reflect the impact of this pathogen in clinical practice and the overall adherence to new recommendations. This change was not associated with an increase in antibiotic resistance. Clindamycin is an alternative treatment although Clindamycin inducible resistance is a worry. Continuous monitoring of antibiotic consumption and local susceptibility patterns are required to promote rational use of antibiotics.

Key words: Staphylococcus aureus, antimicrobial agents, consumption, antimicrobial susceptibility.

Palabras clave: Staphylococcus aureus, consumo antimicrobianos, susceptibilidad antimicrobiana.

\section{Introducción}

$\mathrm{E}$ n la década del 90, se comenzó a observar un aumento internacional en la frecuencia de aislados de Staphylococcus aureus meticilina resistente (SAMR) no multi-resistente en niños y adultos con infecciones adquiridas en la comunidad. Su aparición se ha asociado, no sólo con infecciones de piel y tejidos blandos, sino con infecciones invasoras, con elevada morbi-mortalidad ${ }^{1-4}$.

En Uruguay, los primeros casos fueron reportados en los años 2003 y 2005 5 . . Desde entonces, en el Hospital Pediátrico del Centro Hospitalario Pereira Rossell (HPCHPR), centro de referencia nacional, la frecuencia de hospitalizaciones por infecciones no invasoras e invasoras ocasionadas por dicho microorganismo ha aumentado ${ }^{7-9}$.
Universidad de la República, Montevideo, Uruguay Facultad de Medicina Depto. de Farmacología y Terapéutica (HTO, NSM, LLP, GGL) Cátedra de Bacteriología y Virología (GAR)

Centro Hospitalario Pereira Rossell, Montevideo, Uruguay Departamento de Farmacia (ASG, LNR)

Laboratorio de Microbiología (GAR) Departamento de Pediatría (MCPG)

Instituciones responsables: Universidad de la República, Centro Hospitalario Pereira Rossell, Administración de Servicios de Salud del Estado (ASSE)

Recibido: 31 de julio de 2008 Aceptado: 6 de julio de 2009

Correspondencia a: Héctor Telechea O hmteleor@hotmail.com 
Según datos del Laboratorio de Microbiología de este hospital, el número de aislados de SAMR no multirresistente se triplicó en el período 2001-2006. La mayoría de los aislados provenían de muestras de niños con infecciones de piel y tejidos blandos adquiridas en la comunidad.

Estas nuevas cepas de $S$. aureus contienen información genética diferente y su importancia clínica radica en su capacidad invasora y la ausencia de respuesta a los antibacterianos $\beta$-lactámicos. En forma característica, las infecciones por este agente pueden ocurrir en pacientes previamente sanos, sin los clásicos factores de riesgo descritos en las infecciones hospitalarias por SAMR ${ }^{10-12}$. Pueden producir lesiones piógenas necrosantes en piel, tejido subcutáneo, huesos y pulmones, y se asocian con riesgo elevado de secuelas y muerte ${ }^{3,5,6}$.

A diferencia de las cepas hospitalarias, SAMR adquirido en la comunidad generalmente es susceptible a diferentes antibacterianos no $\beta$-lactámicos. Este patrón de susceptibilidad característico se explica por la presencia del gen mec A, que codifica una proteína de unión a penicilina de baja afinidad (PBP2A). Este gen se encuentra en el locus staphylococcal cassette chromosome tipo IV. La virulencia se relaciona con la producción de una proteína de unión al colágeno (cna) y de una exotoxina, leucocidina de Panton Valentine, responsable de la necrosis tisular asociada a infecciones graves. Estas nuevas cepas están particularmente adaptadas para sobrevivir en el ambiente comunitario co, $^{4,13,14}$.

Un problema adicional es el surgimiento de resistencia a otros antimicrobianos como eritromicina y clindamici$\mathrm{na}^{4}$. La resistencia a eritromicina es codificada por genes erm (erythromycin resistance methylase) que producen modificaciones en el sitio de unión ribosómico. Este sitio blanco es compartido por macrólidos, lincosamidas y estreptograminas. Cuando la resistencia a eritromicina es de tipo constitutiva, existe resistencia cruzada a todos los antimicrobianos del grupo (tipo $\mathrm{MLS}_{\mathrm{B}}$ ) y se detecta por los métodos estándares de laboratorio. Cuando ésta es inducible, la bacteria es resistente a macrólidos pero parece susceptible a lincosamidas y estreptograminas B con el uso de técnicas estándares de evaluación. Para detectar la resistencia inducible a clindamicina es necesario utilizar otras pruebas, como el "D”-test (disco difusión, prueba de inducción) ${ }^{15}$.

En general, el fenómeno "D" se describe como de baja frecuencia (5-10\%). En Uruguay, se desconoce su frecuencia. Más aún, el impacto clínico de este fenómeno todavía no ha sido establecido. Desde el año 2005 las guías del Clinical and Laboratory Standards Institute (CLSI) recomiendan su detección en los laboratorios de Microbiología ${ }^{16}$.

En Uruguay, la emergencia de SAMR adquirido en la comunidad llevó a modificar las recomendaciones de antibioterapia empírica y a implementar un sistema de vigilancia epidemiológica. Las nuevas pautas de tratamiento antibacteriano se comenzaron a aplicar en el año 2004 y están sometidas a evaluación periódica ${ }^{17-20}$.

El siguiente trabajo tiene como objetivo describir la evolución de la susceptibilidad in vitro de $S$. aureus y su relación con el consumo de antimicrobianos en el HPCHPR en el período 2001-2006.

\section{Metodología}

Se estimó el consumo de los antimicrobianos habitualmente recomendados en el tratamiento empírico de infecciones de probable etiología estafilocóccica adquiridas en la comunidad, incluidos en la pauta de tratamiento de infecciones de piel y tejidos blandos, neumonía y osteoartritis (cefradina, cefuroxima, ceftriaxona, clindamicina, cotrimoxazol, vancomicina y gentamicina) en los niños hospitalizados en el HP CHPR en el período 2001-2006.

Las áreas de internación incluidas fueron: pediatría general, unidad de cuidados intensivos, aislamientos, cirugía y ortopedia.

Se utilizó como medida de consumo la dosis diaria definida (DDD) por 100 camas día (DDD/100).

Para su cálculo se aplicó la siguiente fórmula ${ }^{21}$ :

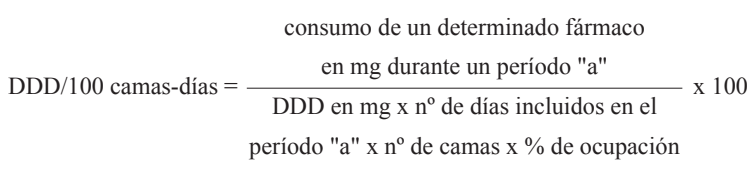

Los datos de consumo se obtuvieron del Programa WinFarma del Departamento de Farmacia del CHPR.

La DDD para cada antimicrobiano se expresó en miligramos y representa la dosis de mantenimiento utilizada para la principal indicación del medicamento. El valor de las DDD se obtuvo del Collaborating Centre for Drug Statistics Methodology de la Organización Mundial de la Salud ${ }^{22}$

El número de camas y el porcentaje de ocupación fueron aportados por la División Estadística del CHPR.

Los datos de susceptibilidad in vitro de $S$. aureus se obtuvieron del Laboratorio de Microbiología del CHPR. Se incluyeron los aislados obtenidos de piel, tejidos blandos y sitios normalmente estériles de los pacientes hospitalizados en el HP-CHPR en el período de estudio. Los patrones de susceptibilidad in vitro se estudiaron mediante el método de difusión en disco en agar siguiendo los lineamientos del CLSI ${ }^{16}$. Los antimicrobianos probados fueron: oxacilina, eritromicina, clindamicina, cotrimoxazol y gentamicina. El fenómeno de resistencia inducida a clindamicina se vigila en el laboratorio desde el año 2004 por "D"-test. 
La evolución de las DDD y de la susceptibilidad antimicrobiana se expresó en porcentaje. Se calculó el cambio porcentual en las DDD 2001-2006. Se consideró una diferencia estadísticamente significativa $\mathrm{p} \leq 0,05$. Para el procesamiento de los datos se utilizó el programa Epi info 6.0.

\section{Resultados}

En el período de estudio se observó un aumento significativo en el consumo de todos los antimicrobianos analizados, excepto cefradina que disminuyó. La evolución de las DDD/100 se muestra en la Tabla 1. Los antimicrobianos que presentaron mayor aumento en su consumo fueron clindamicina, ceftriaxona y cotrimoxazol. En los años 2001 y 2002 el consumo de clindamicina oral e intravenosa fue escaso. A partir de 2003, éste aumentó en forma significativa manteniéndose elevado hasta el año 2006. El aumento porcentual fue $1.718 \%$ y $6.150 \%$ para las formulaciones intravenosa y oral, respectivamente. Los aumentos porcentuales en el consumo de vancomicina, cefuroxima y gentamicina fueron menores que los de clindamicina, ceftriaxona y cotrimoxazol.

A lo largo del período de estudio se observó un aumento en el número de aislados de $S$. aureus. El 93,1\% (n: 6.025) de las cepas fueron obtenidas de muestras de piel y tejidos blandos; $4,1 \%$ (n: 268 ) de sangre y $2,7 \%$ (n: 178) de tejido óseo y/o líquido articular. En la Tabla 2 se muestra la evolución de la susceptibilidad in vitro a los antimicrobianos de todas las cepas aisladas $(\mathrm{n}=$ 6.471). A partir del año 2003 se observó una disminución significativa y mantenida de la susceptibilidad a oxacilina. La susceptibilidad a clindamicina, cotrimoxazol y gentamicina se mantuvo por encima de $80 \%$ durante todo el período. Si bien la susceptibilidad a eritromicina fue más baja, no disminuyó. Entre los años 2004 y 2006 el efecto D disminuyó de 28 a $21 \%$.

En las Tablas 3, 4 y 5 se muestra la evolución de la

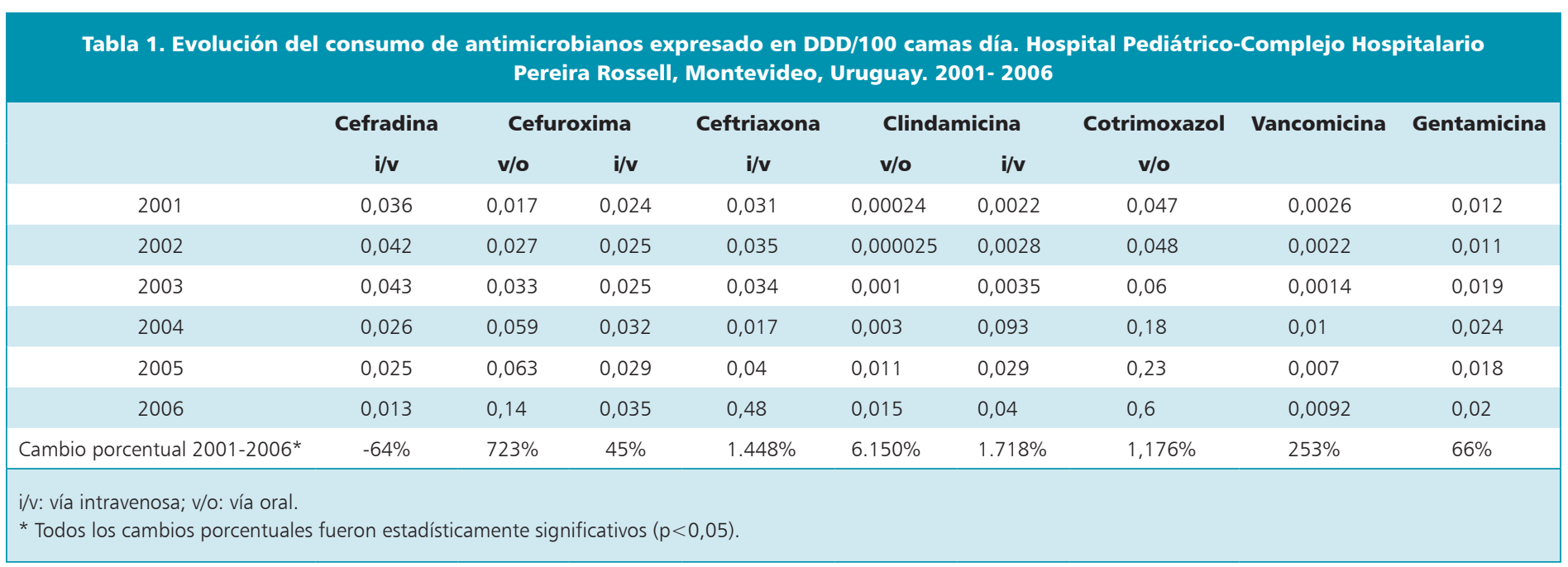

\begin{tabular}{|c|c|c|c|c|c|c|c|c|c|c|c|c|c|c|}
\hline \multirow[b]{2}{*}{ Oxacilina } & \multirow[b]{2}{*}{ n (\%) } & \multicolumn{2}{|c|}{$\begin{array}{c}2001 \\
(n=499)\end{array}$} & \multicolumn{2}{|c|}{$\begin{array}{c}2002 \\
(n=486)\end{array}$} & \multicolumn{2}{|c|}{$\begin{array}{c}2003 \\
(n=735)\end{array}$} & \multicolumn{2}{|c|}{$\begin{array}{c}2004 \\
(n=1.752)\end{array}$} & \multicolumn{2}{|c|}{$\begin{array}{c}2005 \\
(n=1.746)\end{array}$} & \multicolumn{2}{|c|}{$\begin{array}{c}2006 \\
(n=1.253)\end{array}$} & \multirow{2}{*}{ 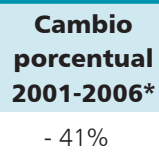 } \\
\hline & & 404 & $(81 \%)$ & 364 & $(75 \%)$ & 374 & $(51 \%)$ & 665 & (38\%) & 715 & $(41 \%)$ & 501 & $(40 \%)$ & \\
\hline Eritromicina & n (\%) & 329 & $(66 \%)$ & 291 & $(60 \%)$ & 418 & $(57 \%)$ & 1.121 & $(64 \%)$ & 1.204 & $(69 \%)$ & 914 & $(73 \%)$ & $7 \%$ \\
\hline Clindamicina & n (\%) & 409 & $(82 \%)$ & 456 & (94\%) & 676 & $(92 \%)$ & 1.699 & $(97 \%)$ & 1.728 & (99\%) & 1.227 & $(98 \%)$ & $16 \%$ \\
\hline Cotrimoxazol & n (\%) & 419 & $(84 \%)$ & 466 & $(96 \%)$ & 683 & (93\%) & 1.716 & (98\%) & 1.728 & (99\%) & 1.240 & (99\%) & $15 \%$ \\
\hline Gentamicina & n (\%) & 414 & $(83 \%)$ & 452 & $(93 \%)$ & 676 & $(92 \%)$ & 1.699 & $(97 \%)$ & 1.728 & (99\%) & 1.215 & $(97 \%)$ & $14 \%$ \\
\hline Efecto-D & n (\%) & & - & & - & & - & 490 & (28\%) & 471 & $(27 \%)$ & 263 & $(21 \%)$ & $-7 \%$ \\
\hline
\end{tabular}




\begin{tabular}{|c|c|c|c|c|c|c|c|c|}
\hline & & $\stackrel{2}{(n=}$ & $\begin{array}{l}001 \\
=423)\end{array}$ & $\begin{array}{c}2002 \\
(n=402)\end{array}$ & $\begin{array}{c}2003 \\
(n=651)\end{array}$ & $\begin{array}{c}2004 \\
(n=1.647)\end{array}$ & $\begin{array}{c}2005 \\
\text { (n }=1.691)\end{array}$ & $\begin{array}{c}2006^{*} \\
(n=1.203)\end{array}$ \\
\hline Oxacilina & n (\%) & 342 & $(81 \%)$ & 297 (74\%) & 331 (51\%) & 617 (37\%) & $683(40 \%)$ & 476 (39\%) \\
\hline Eritromicina & $\mathrm{n}(\%)$ & 278 & $(66 \%)$ & 246 (61\%) & $380 \quad(58 \%)$ & 1.064 (65\%) & $1.173(69 \%)$ & $876(73 \%)$ \\
\hline Clindamicina & n (\%) & 348 & $(82 \%)$ & 378 (94\%) & 603 (93\%) & 1.611 (98\%) & 1.673 (99\%) & $1.178(98 \%)$ \\
\hline Cotrimoxazol & $n(\%)$ & 354 & $(84 \%)$ & 388 (96\%) & 608 (93\%) & $1.716(98 \%)$ & 1.671 (99\%) & $1.190(99 \%)$ \\
\hline Gentamicina & $n(\%)$ & 349 & $(82 \%)$ & 374 (93\%) & 601 (92\%) & 1.615 (98\%) & 1.671 (99\%) & 1.167 (97\%) \\
\hline Efecto-D & n (\%) & & - & - & - & $460(28 \%)$ & 453 (27\%) & 245 (20\%) \\
\hline
\end{tabular}

\begin{tabular}{|c|c|c|c|c|c|c|c|c|c|c|c|}
\hline \multirow[b]{2}{*}{ Oxacilina } & \multirow[b]{2}{*}{ n (\%) } & $\begin{array}{c}2001 \\
(n=21)\end{array}$ & \multicolumn{2}{|c|}{$\begin{array}{c}2002 \\
(n=32)\end{array}$} & \multicolumn{2}{|c|}{$\begin{array}{c}2003 \\
(n=31)\end{array}$} & \multicolumn{2}{|c|}{$\begin{array}{c}2004 \\
(n=43)\end{array}$} & \multicolumn{2}{|c|}{$\begin{array}{c}2005 \\
(n=30)\end{array}$} & $\begin{array}{c}2006 \\
(n=31)\end{array}$ \\
\hline & & $21 \quad(100 \%)$ & 25 & $(80 \%)$ & 14 & $(45 \%)$ & 20 & $(47 \%)$ & 13 & $(43 \%)$ & $16 \quad(52 \%)^{*}$ \\
\hline Eritromicina & n (\%) & $16 \quad(76 \%)$ & 17 & $(55 \%)$ & 9 & $(29 \%)$ & 20 & $(47 \%)$ & 12 & $(40 \%)$ & $22 \quad(71 \%)$ \\
\hline Clindamicina & $n(\%)$ & $21 \quad(100 \%)$ & 29 & $(93 \%)$ & 28 & $(90 \%)$ & 33 & (77\%) & 28 & $(93 \%)$ & $31 \quad(100 \%)$ \\
\hline Cotrimoxazol & n (\%) & $21 \quad(100 \%)$ & 29 & $(93 \%)$ & 30 & $(97 \%)$ & 42 & (98\%) & 30 & $(100 \%)$ & $31 \quad(100 \%)$ \\
\hline Gentamicina & n (\%) & $21 \quad(100 \%)$ & 29 & $(93 \%)$ & 30 & $(97 \%)$ & 36 & $(84 \%)$ & 30 & $(100 \%)$ & $30 \quad(97 \%)$ \\
\hline Efecto-D & n (\%) & - & & - & & - & 17 & $(40 \%)$ & 11 & (37\%) & $9 \quad(29 \%)$ \\
\hline
\end{tabular}

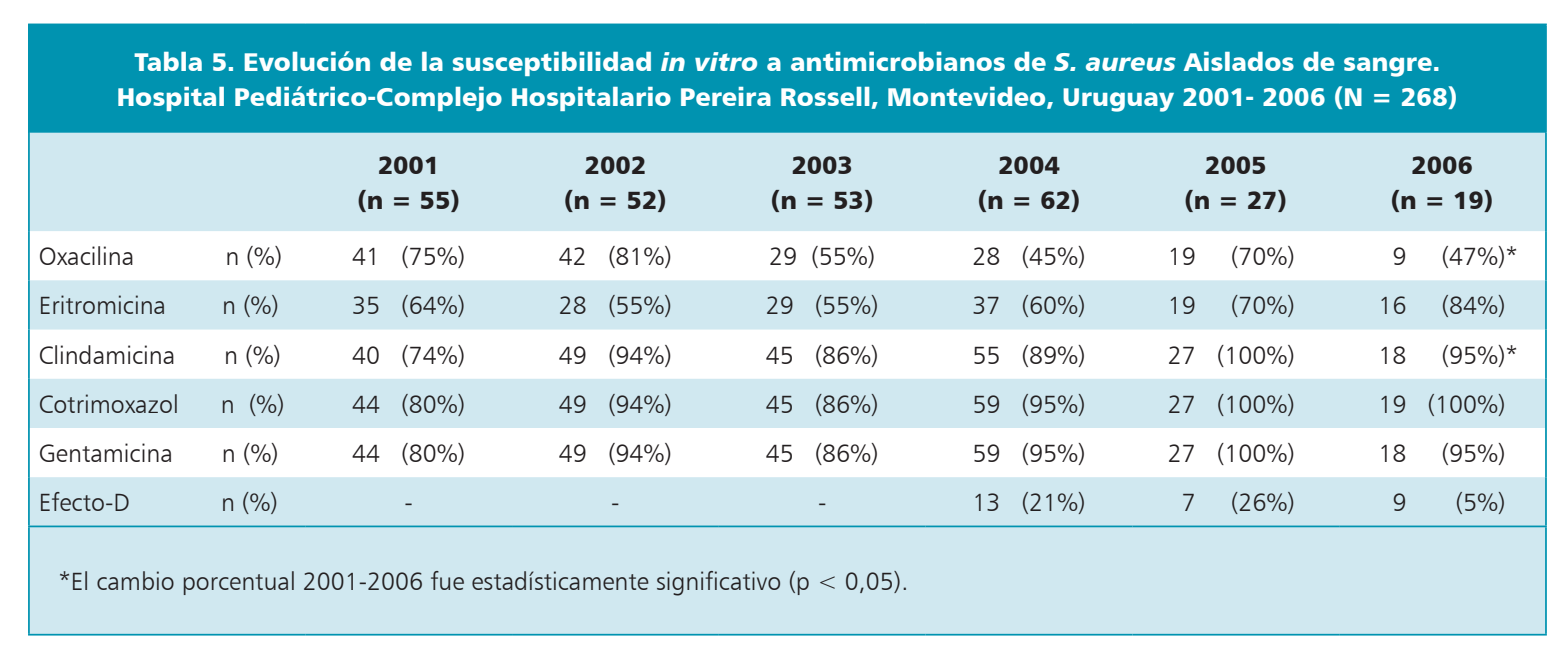


susceptibilidad antimicrobiana de los aislados obtenidos a partir de infecciones de piel y tejidos blandos, osteoarticulares y sangre, respectivamente. No se observaron diferencias significativas en el patrón de susceptibilidad a los antimicrobianos estudiados en función de la fuente de aislamiento, excepto en el fenómeno " $\mathrm{D}$ ” que fue más frecuente en los aislados osteo-articulares, si bien tampoco tuvo significación estadística.

\section{Discusión}

En este estudio se describe un aumento en el consumo de ciertos antimicrobianos en relación con un cambio significativo en el patrón de susceptibilidad in vitro de $S$. aureus. Si bien existe una relación entre estas dos variables, la misma no es necesariamente de tipo causal. No es posible predecir qué ocurrirá con la susceptibilidad in vitro a antimicrobianos a partir de los cambios en su consumo, ya que éste no es el único factor que interviene.

A pesar de esto, el análisis de estos datos permite afirmar que SAMR no multiresistente se instaló definitivamente como patógeno en las infecciones del niño adquiridas en la comunidad. En este contexto epidemiológico, la vigilancia del uso de antimicrobianos constituye una estrategia fundamental.

$\mathrm{Si}$ bien este estudio tiene algunas limitaciones derivadas del método utilizado para evaluar el consumo de antimicrobianos, los resultados permiten una buena caracterización de los hábitos de prescripción desde el surgimiento del SAMR no multi-resistente. La DDD es una unidad técnica internacional que permite comparar el consumo de medicamentos en distintas regiones y distintos períodos, en forma independiente de las variaciones de precio y de las especialidades farmacéuticas. Es un indicador de cambios en los hábitos terapéuticos, si bien no identifica las causas específicas de éstos. La DDD/100 es la unidad utilizada para evaluar el consumo a nivel hospitalario. En su cálculo se utilizan datos de dispensación, y se asume que ésta refleja el consumo y éste a su vez, la prescripción. Por tanto, puede modificarse por variaciones en la frecuencia de la enfermedad y en los hábitos de prescripción de los profesionales ${ }^{21}$.

Es posible que los cambios observados en el consumo de antimicrobianos estén asociados con la implementación de las nuevas recomendaciones de antibioterapia empírica y con un alto grado de cumplimiento de las mismas.

Las infecciones de piel y tejidos blandos constituyen, por su elevada frecuencia, una de las principales indicaciones de estos antibacterianos. En el tratamiento de estas infecciones es donde las recomendaciones terapéuticas incluyeron los cambios más importantes. Previamente se recomendaba el uso sistemático de cefradina como antibiótico de primera línea, y actualmente se recomienda su administración combinada con gentamicina o el uso alternativo de cotrimoxazol o clindamicina ${ }^{18,19}$. La elección depende, de la presencia de elementos clínicos que orienten a la etiología estafilocóccica (impétigo buloso, forúnculos, abscesos, celulitis graves). En todos los casos es necesario, además, implementar un tratamiento local. Habitualmente se recomienda el uso combinado de antimicrobianos por vía sistémica y tópica, y el lavado, descostrado o drenaje de las lesiones. En este estudio no se analizó el consumo de los antimicrobianos tópicos utilizados con mayor frecuencia, como mupirocina o ácido fusídico. No se dispone de datos sobre los patrones de uso ni de susceptibilidad in vitro a éstos. Es necesario, junto a los microbiólogos, implementar un programa de vigilancia que los incluya.

El elevado consumo de clindamicina oral e intravenoso puede obedecer a que este antibacteriano constituye una alternativa terapéutica para el tratamiento empírico de infecciones graves de piel y tejidos blandos, neumonía e infecciones osteo-articulares. Clindamicina ofrece un espectro de actividad adecuado para estafilococos y estreptococos, incluido Streptococcus pneumoniae. La falta de formulaciones farmacéuticas apropiadas para niños pequeños y lactantes en nuestro medio no limitó su uso extendido. Debido a que cotrimoxazol carece de actividad apropiada frente a estreptococos, su uso empírico no se recomienda en aquellas situaciones en las que no existe clara orientación etiológica. Esto podría explicar el menor aumento en el consumo observado de este antibacteriano respecto a clindamicina.

Al igual que lo descrito en otras regiones, en nuestro medio se observa resistencia inducible a clindamicina. Este fenómeno fue más frecuente que lo descrito en la literatura, especialmente en aislados de infecciones osteo-articulares ${ }^{23}$; constituye un fenómeno preocupante ante el aumento significativo en la prescripción de clindamicina. Su importancia clínica no ha sido completamente establecida. Es necesario realizar estudios prospectivos que relacionen el fenómeno " $D$ " con la evolución clínica. La disminución observada en el fenómeno D a pesar del aumento significativo en el consumo de clindamicina es un hecho llamativo. Se desconoce el significado de este hallazgo.

El aumento observado en el consumo de ceftriaxona puede estar relacionado con su uso en el tratamiento empírico del paciente con infecciones graves. Las recomendaciones la incluyen en forma combinada a vancomicina en el tratamiento del niño grave con neumonía, de modo de cubrir $S$. pneumoniae resistente a penicilina y otros microorganismos menos habituales.

Es necesario evaluar el impacto de los cambios observados en el consumo de clindamicina, cotrimoxazol y ceftriaxona sobre el desarrollo de resistencia en microorganismos adquiridos, tanto en la comunidad como 
en el hospital, y en la frecuencia de los efectos adversos característicos de estos antimicrobianos.

En esta era de SAMR, el aumento del consumo de vancomicina es un hecho esperable. A pesar de esto, su aumento porcentual fue inferior al de otros antibacterianos. En algunas regiones del mundo se ha descrito el surgimiento de cepas de $S$. aureus con susceptibilidad disminuida a este antimicrobiano, fenómeno no registrado aún en nuestro medio. Es preocupante que, en un estudio previo que evaluó las características del uso de vancomicina en el HP CHPR, se identificaron problemas relacionados con la selección y la duración del tratamiento con vancomicina, que alertaron sobre un posible uso inadecuado ${ }^{24}$. En función de estos hallazgos, es necesario continuar controlando el uso de vancomicina a nivel hospitalario y promover el cumplimiento de las recomendaciones entregadas por el Centers for Disease Control and Prevention (CDC) de E.U.A. para prevenir la diseminación de la resistencia a este antibacteriano ${ }^{25,26}$.

Es importante una vigilancia sistemática del uso de antimicrobianos como una forma de promover su uso racional y detener el desarrollo de resistencia. Este tipo de estudios constituye un método "sensible", rápido y barato para la detección de problemas en la prescripción, que sirvan de base para investigar sus posibles causas. La vigilancia permanente de la susceptibilidad antimicrobiana in vitro aporta información indispensable para la actualización de las recomendaciones vigentes. Este tipo de actividades son promovidas en el "Programa de control de la resistencia antimicrobiana" de la Organización Panamericana de la Salud/Organización Mundial de la Salud (OPS/OMS) y se enmarcan en el Programa de Uso Racional de Medicamentos del HP-CHPR ${ }^{27,28}$.

\section{Conclusiones}

A pesar que no es posible establecer una relación causal entre consumo y susceptibilidad a antimicrobianos, los resultados de este estudio permiten concluir que, en Uruguay, SAMR no multi-resistente se ha establecido como un patógeno emergente.

La susceptibilidad a antimicrobianos hallada muestra la vigencia de las recomendaciones de antibioterapia empírica para el tratamiento de las infecciones de piel y tejidos blandos, neumonía, e infecciones osteo-articulares, elaboradas en el año 2004.
El aumento observado en el consumo de aquellos antimicrobianos con adecuado espectro frente a SAMR no multi-resistente probablemente se relacione con la aceptación y cumplimiento de estas recomendaciones.

Es necesario mantener una vigilancia periódica de los posibles cambios en la susceptibilidad in vitro a estos antibacterianos, con especial énfasis en la aparición de resistencia inducible a clindamicina y su importancia clínica.

La monitorización del consumo de antimicrobianos y el análisis de la epidemiología local resultan indispensables para promover su uso racional.

\section{Resumen}

Introducción: En Uruguay, Staphylococcus aureus resistente a meticilina no multi-resistente se instaló como patógeno en las infecciones comunitarias del niño. Objetivo: Describir la evolución de la susceptibilidad in vitro de $S$. aureus y su relación con el consumo de antimicrobianos en el Centro Hospitalario Pereira Rossell (CHPR) entre 2001-2006. Metodología: Se estimó el consumo de antimicrobianos mediante el cálculo de la Dosis Diaria Definida por 100 camas-día. Los valores de referencia fueron obtenidos del Collaborating Centre for Drug Statistics Methodology of World Health Organization. Los datos de consumo fueron extraídos del programa WinFarma del Dpto de Farmacia de CHPR. El porcentaje de ocupación de camas/año se obtuvo de la División de Estadísticas del CHPR. Se analizó la susceptibilidad antimicrobiana de $S$. aureus de aislados de piel, tejidos blandos y sitios normalmente estériles. Se analizó la evolución del consumo y la susceptibilidad in vitro en el período 2001-2006. Resultados: Desde el año 2003, el consumo de clindamicina, ceftriaxona y cotrimoxazol aumentó y la susceptibilidad a oxacilina disminuyó significativamente. Entre 2004 y 2006 el “efecto D” disminuyó su frecuencia desde 28 a $21 \%$. No se observaron diferencias en el patrón de susceptibilidad antimicrobiana en función del sitio de aislamiento. Conclusiones: Staphylococcus aureus resistente a meticilina no multi-resistente se ha introducido en forma significativa como un patógeno causante de infecciones en la comunidad en niños uruguayos. La vigilancia del consumo de antimicrobianos y de la epidemiología local es indispensable para actualizar las guías de antibioterapia empírica. 


\section{Referencias}

1.- Collignon P, Gosbell I, Vickery A, Nimmo G, Stylianopoulos T, Gottlieb T, et al. Communityacquired methicillin-resistant Staphylococcus aureus in Australia. Lancet 1998; 352: 145-6.

2.- Fergie J, Purcell K. Community-acquired methicillin-resistant Staphylococcus aureus infections in South Texas children. Pediatr Infect Dis J 2001; 20: 860-3.

3.- An Diep B, Sensabaugh G, Somboona N, Carleton H, Perdreau-Remington F. Widespread skin and soft-tissue infections due to two methicillin-resistant Staphylococcus aureus strains harboring the genes for Panton-Valentine leucocidin. J Clin Microbiol 2004; 42 (5): 2080-4.

4.- Vandenesch F, Naimi T, Enright M, Lina G, Nimmo G, Heffernan H, et al. Communityacquiered methicillin-resistant Staphylococcus aureus carring Panton-Valentine leukocidin genes: Worldwide emergence. Emerg Infect Dis 2003; 9 (8): 978-84.

5.- Galiana A. Infección por Staphylococcus aureus meticilino resistente adquirido en la comunidad. Arch Pediatr Urug 2003; 74 (1): 26-9.

6.- Xue Ma X, Galiana A, Pedreira W, Mowszowicz M, Christophersen I, Machiavello S, et al. Community-acquired methicillin-resistant Staphylococcus aureus, Uruguay. Emerg Infect Dis 2005; 11 (6): 973-6.

7.- Prego J, Galiana A, Pujadas M, Almada K, Boulay M, Carugatti M. Infecciones de piel y partes blandas en pacientes ambulatorios. Arch Pediatr Urug 2004; 75 (4): 300-6.

8.- Parodi V, Aldao J, Bustos R. Estafilococo meticilino resistente en la maternidad del Centro Hospitalario Pereira Rossell. Arch Pediatr Urug 2007; 78 (1): 5-10.

9.- Amorín M, Castro M, Sandín D, Chamorrro F, Romero C, Giachetto G, et al. Infecciones invasivas por Staphylococcus aureus meticilino resistente adquirido en la comunidad. Presentación clínica y evolutiva observada en dos centros universitarios. Uruguay 2003-2007. Rev Med Urug 2008; 24: 230-7

10.- Chamorro F, Grierson C, Martínez C, Laurito G, Matta A, Sandin D, et al. Factores de riesgo de infección por Staphylococcus aureus meticilino resistente en niños con infecciones adquiridas en la comunidad, asistidos en el Centro Hospitalario Pereira Rossell. Presentado en: II Congreso de Pediatría ambulatoria. Montevideo, Uruguay 2004. (falta ubicar en libro de resúmenes: página y número de resumen)

11.- Herold B, Immergluck L, Maranan M, Lauderdale D, Gaskin R, Boyle-Vavra S, et al. Community-acquired methicillin-resistant Staphylococcus aureus in children with no identified predisposing risk. JAMA 1998; 279: 593-8

12.- Sattler C, Mason E, Kaplan S. Prospective comparison of risk factors and demographic and clinical characteristics of community-acquired, methicillin-resistant versus methicillin-susceptible Staphylococcus aureus infection in children. Pediatr Infect Dis J 2002; 21: 910-7.

13.- Dufour P, Gillet Y, Bes M, Lina G, Vandenesch F, Floret D, et al. Communityacquired methicillin-resistant Staphylococcus aureus infections in France: emergence of a single clone that produces Panton-Valentine leukocidin. Clin Infect Dis 2002; 35: 819-24.

14.- Lina G, Pièmont $Y$, Godail-Gamot F, Bes M, Peter M, Gauduchon V, et al. Involvement of Panton-Valentine leukocidin-producing Staphylococcus aureus in primary skin infections and pneumonia. Clin Infect Dis 1999; 29: 1128-32

15.- Opal SM, Medeiros AA. Molecular mechanisms of antibiotic resistance in bacteria. En: Mandell, Douglas and Bennett's Principles and Practice of Infectious Diseases, Mandell GL, Bennett JE \& Dolin R, eds.: 6th ed. 2005. Churchill Livingstone, Elsevier, Philadelphia, pp 253-270.

16.- Clinical and Laboratory Standards Institute. 2005. Performance standards for antimicrobial susceptibility testing; fifteenth informational supplement, M100-S15. Clinical and Laboratory Standards Institute. Wayne (PA), USA.

17.- Neumonía. En: Atención Pediátrica. Pautas de diagnóstico, tratamiento y prevención. $6^{a}$ ed. 2007. Montevideo: Oficina del libro. AEM, pp 161-9.

18.- Impétigo. Abscesos y forúnculos. En: Atención Pediátrica. Pautas de diagnóstico, tratamiento y prevención. $6^{\mathrm{a}}$ ed. 2007, Montevideo: Oficina del libro. AEM, pp 301-5.
19.- Celulitis. En: Atención Pediátrica. Pautas de diagnóstico, tratamiento y prevención. $6^{\mathrm{a}}$ ed 2007. Montevideo: Oficina del libro. AEM, pp 309-12

20.- Osteomielitis aguda. Artritis aguda. En: Atención Pediátrica. Pautas de diagnóstico, tratamiento y prevención. $6^{a}$ ed. 2007. Montevideo: Oficina del libro. AEM, pp 313-8.

21.- Capella D, Laporte J. Métodos aplicados en estudios descriptivos de utilización de medicamentos. En: Laporte J,Tognoni G. Principios de epidemiología del medicamento. 2ed. 2003. Barcelona: Masson-Salvat, pp 67-93.

22.- Who Collaborating Centre for Drugs Statistics Methodology. Obtenido: http://www.whocc.no/ atcddd/ (falta fecha de acceso)

23.- Szczesiul J, Shermock K, Murtaza U, Siberry G. No decrease in clindamycin susceptibility despite increased use of clindamycin for pediatric community-associated methicillin-resistant Staphylococcus aureus skin infections. Pediatr Infect Dis J 2007: 26 (9): 852-4.

24.- Giachetto G, Telechea H, Speranza N, Andruskevicius M, Salazar S, Aramendi I, et al. Uso de vancomicina en servicios de internación pediátrica del Centro Hospitalario Pereira Rossell. Arch Pediatr Urug 2006; 77 (2): 118-24.

25.- Centers for Disease Control and Prevention. Recommendation for preventing the spread of vancomycin resistance: Recommendation of the Hospital Infection Control Practices Advisory Committee. MMWR Morb Mortal Wkly Rep 1995; 44 (RR-12): 1-13.

26.- Keyserling H, Sinkowitz-Cochran R, Harris J, Levine G, Siegel J, Stover B, et al. Vancomycin use in hospitalized pediatric patients. Pediatrics 2003; 112: 104-11.

27.- World Health Organization. Containing antimicrobial resistance: review of the literature and report of a WHO Workshop on the Development of a Global Strategy for the Containment of Antimicrobial Resistance. Geneva: WHO, 1999: 1-54.

28.- Giachetto G, Martínez A, Pírez M, Algorta G, Banchero P, Camacho G, et al. Vigilancia del uso de antibióticos en el Hospital Pediátrico del Centro Hospitalario Pereira Rossell. Rev Med Uruguay 2003; 19: 208-15. 